\title{
The Random Walk and Trend Stationary Models with an Analysis of the US Real GDP: Can We Distinguish between the Two Models?
}

\author{
Kazumitsu Nawata1,2 \\ ${ }^{1}$ Graduate School of Engineering, University of Tokyo, Bunkyo-ku, Tokyo, Japan \\ ${ }^{2}$ Research Center for Health Policy and Economics, Hitotsubashi Institute for Advanced Study, Hitotsubashi University, \\ Kunitachi-shi, Tokyo, Japan \\ Email:nawata@tmi.t.u-tokyo.ac.jp
}

How to cite this paper: Nawata, K. (2021) The Random Walk and Trend Stationary Models with an Analysis of the US Real GDP: Can We Distinguish between the Two Models? Open Journal of Statistics, 11, 213-229.

https://doi.org/10.4236/ojs.2021.111011

Received: December 10, 2020

Accepted: February 23, 2021

Published: February 26, 2021

Copyright $\odot 2021$ by author(s) and Scientific Research Publishing Inc. This work is licensed under the Creative Commons Attribution International License (CC BY 4.0).

http://creativecommons.org/licenses/by/4.0/

\begin{abstract}
The unit root can lead to major problems in economic time series analyses. I obtain the asymptotic distributions of the ordinary least squares (OLS) estimator when the true model is trend stationary for the following three cases: 1) the null model is a random walk without drift, and the auxiliary regression model does not contain a constant; 2) the null model is a random walk with drift, and the auxiliary regression model contains a constant; and 3) the null model is a random walk with drift, and the auxiliary regression model contains both a constant and a time trend. In the third case, the asymptotic distribution of the OLS estimator is determined by the first order of the autocorrelation, and we can distinguish between the random walk and trend stationary models, unlike in previous studies. Based on these results, the real US gross domestic product is analyzed. A time trend model with autoregressive error terms is chosen. The results suggest that the impacts of a shock can become larger than the original shock in some periods and then gradually decline. However, the impacts continue for a long period, and policy makers should account for this to design better economic policies.
\end{abstract}

\section{Keywords}

Dickey-Fuller Test, Unit Root, Random Walk, Trend Stationary, US GDP

\section{Introduction}

The unit root can lead to major problems in economic time series analyses. The Dickey-Fuller (DF) test [1] is one of the most widely used unit root tests. Various models, alternatives, and testing procedures for both the DF and the aug- 
mented DF (ADF) [2] tests have been studied [3]-[10]. It has been reported that the power of the DF test is low when the alternative model is the trend stationary model, which is one of the most important alternatives to the random walk model. As Enders [[11], p. 235] wrote, "[t]he problem is that, in finite samples, any trend stationary process can be arbitrarily well approximated by a unit root process, and a unit root process can be arbitrarily approximated by a trend stationary process". In addition, as Haldrup and Jansen [[12], p. 261] wrote, "[f]or instance, failure to include a time trend regressor in auxiliary regression when power against the trend stationary alternative is wanted will lead to a test with zero asymptotic power". However, neither of these studies expressly show the asymptotic distributions of the ordinary least squares (OLS) estimator in the trend stationary case. West [13] considered the asymptotic distributions of OLS estimator and concluded that the asymptotic power of the DF test is zero when the alternative model is trend stationary. However, his results were rather incomplete. The asymptotic distributions of the OLS estimator and the behavior of the DF test depend on the true, null, alternative, and auxiliary regression models used in the test. To improve the tests, it is necessary to obtain the asymptotic distributions of the OLS estimator when the true model is trend stationary and combine them with the results of the traditional DF tests.

In this paper, following Schmidt and Phillips [14], I consider the asymptotic distributions of the OLS estimator when the true model is trend stationary in the following three cases: 1) the null model is a random walk without drift, and the auxiliary regression model does not contain a constant; 2) the null model is a random walk with drift, and the auxiliary regression model contains a constant; and 3) the null model is a random walk with drift and the auxiliary regression model contains both a constant and a time trend.

Based on these results, I analyze the real US gross domestic product (GDP). The problem of whether GDP is a random walk or trend stationary has been studied by various authors [15]-[23]. The results have been mixed; some found that GDP was a random walk (nonstationary) and others found that GDP was (trend) stationary. The real US GDP data from the first quarter of 1948 to the fourth quarter of 2019 , i.e., 280 quarters or 70 years, are analyzed in the present study. Finally, I simulate the impacts of economic shocks using the obtained trend stationary model with autoregressive (AR) process errors.

\section{Models and Asymptotic Distributions of the OLS Estimator}

In this section, I obtained the asymptotic distributions of the OLS estimator for the three cases when the true model is the trend stationary model, given by

$$
y_{t}=y_{0}+\alpha t+u_{t}, u_{t}=\varepsilon_{t}+\phi_{1} \varepsilon_{t-1}+\phi_{2} \varepsilon_{t-2}+\cdots+\phi_{q} \varepsilon_{t-q}, t=1,2, \cdots, T .
$$

where $\varepsilon_{1}, \varepsilon_{2}, \cdots, \varepsilon_{T}$ are independent and identically distributed (i.i.d.) random variables following $N\left(0, \sigma^{2}\right)$. Let $m_{s}=E\left(u_{t} u_{t-s}\right)=\sum_{k=1}^{q} \phi_{k} \phi_{k-s} \sigma^{2}$ where $\phi_{0}=1$ and $\phi_{j}=0$ if $j<0$. Then $m_{0}=V\left(u_{t}\right)$. The value of $q$ may be infinite, but the following conditions are satisfied: 


$$
\sum_{s=1}^{q}\left|m_{s}\right| s^{2}<\infty \text { and }
$$

$\sum_{j=1}^{q} E\left(\xi_{i} \xi_{j}\right)<\infty$ for any $i$, where $\xi_{i}=u_{i} u_{i-1}-m_{1}$.

These conditions are satisfied if $q$ is finite or $u_{t}$ is a stationary AR process. It is also assumed that the fourth moment of $u_{t}$ is finite. Phillips and Perron [24] assumed similar conditions for the error terms for the random walk model; however, I assume these conditions for the trend stationary model.

I first consider the case in which the null model is a random walk without drift. Secondly, I analyze the case in which the null model is a random walk with drift and the auxiliary regression model contains a constant. Finally, I consider the case in which the auxiliary regression model contains both a constant and a time trend.

\subsection{Random Walk Model and Auxiliary Regression Model without a Constant}

I consider the case in which the null hypothesis is the random walk model given by

$$
H_{0}: y_{t}=y_{t-1}+\varepsilon_{t}, t=1,2, \cdots, T .
$$

We consider the auxiliary regression model,

$$
y_{t}=\rho y_{t-1}+\omega_{t}, y_{0}=0 \text { and } \omega_{0}=0, t=1,2, \cdots, T \text {. }
$$

Long and Herrera [25] considered the asymptotic properties of the OLS estimator of the trend stationary model when the true model is a random walk; however, we usually use the OLS estimator (4) for the unit root tests. Note that when $|\rho|<1$, the model becomes trend stationary and is included as a term in (1). The OLS estimator of $\rho$ is given by

$$
\hat{\rho}=\frac{\sum_{t=1}^{T} y_{t} y_{t-1}}{\sum_{t=1}^{T} y_{t-1}^{2}}
$$

Under the null hypothesis, it is well known that (Hamilton [[26], pp. 488-489])

$$
T(\hat{\rho}-1) \stackrel{D}{\longrightarrow} \frac{[W(1)]^{2}-1}{2 \int[W(r)]^{2}} \text { and } \tau=(\hat{\rho}-1) / \text { s.e. }(\hat{\rho}) \stackrel{D}{\longrightarrow} \frac{[W(1)]^{2}-1}{2 \int[W(r)]^{2}}
$$

where $\int=\int_{0}^{1}, W(\cdot)$ represents standard Brownian motion, and s.e. $(\hat{\rho})$ is the standard error.

When the true model is trend stationary as given by (1), the asymptotic distribution of the OLS estimator is given by the following theorem.

Theorem 2.1

When (1) is the true model, the OLS estimator becomes

$$
T(\tilde{\rho}-1) \stackrel{P}{\longrightarrow} \frac{3}{2} \text {. }
$$

The proof is given in Appendix A1. Although the OLS estimator is given by the same formula, I use $\hat{\rho}$ under the null (random walk) model and $\tilde{\rho}$ for the true (trend stationary) model to distinguish the distributions of the estimator. 


\subsection{Random Walk Model with Drift and Auxiliary Regression Model with a Constant}

When we perform unit root tests, it may be more reasonable to consider the random walk model with drift.

Here, the null hypothesis is given by

$$
H_{0}: y_{t}=\alpha+y_{t-1}+\varepsilon_{t}, t=1,2, \cdots, T .
$$

The auxiliary regression model is given by

$$
y_{t}=\alpha+\rho y_{t-1}+\omega_{t}, t=1,2, \cdots, T .
$$

The regression model contains a constant term, and we do not need the initial condition, unlike in (4). As described above, note that when $|\rho|<1$, the model becomes trend stationary and included as a term in (1). The OLS estimator of $\rho$ is given by

$$
\hat{\rho}=\frac{\sum_{t=1}^{T}\left(y_{t-1}-\bar{y}_{1}\right)\left(y_{t}-\bar{y}_{2}\right)}{\sum_{t=1}^{T}\left(y_{t-1}-\bar{y}_{1}\right)^{2}}, \bar{y}_{1}=\frac{\sum_{t=1}^{T} y_{t-1}}{T} \text { and } \bar{y}=\frac{\sum_{t=1}^{T} y_{t}}{T} \text {. }
$$

Under the null hypothesis, the asymptotic distribution is given by (Hamilton [[26], p. 492]),

$$
T^{3 / 2}(\hat{\rho}-1) \stackrel{D}{\longrightarrow} N\left(0,12 m_{0} / \alpha^{2}\right)
$$

When the true model is the trend stationary model given by (1), the asymptotic distribution of the OLS estimator is given by the following theorem.

Theorem 2.2

When (1) is the true model, the OLS estimator (9) becomes

$$
T^{2}(\tilde{\rho}-1) \stackrel{D}{\longrightarrow} N\left(\frac{12}{\alpha^{2}} m_{0}, \frac{36}{\alpha^{2}} m_{0}\right) .
$$

The proof is given in Appendix A2. Theorem 2.2 means that the DF test based on the OLS estimator has no asymptotic power against the trend stationary alternatives as suggested by the previous studies. West [13] showed that $T(\tilde{\rho}-1) \stackrel{P}{\longrightarrow} 0$ but $(\hat{\rho}-1)=O_{P}\left(T^{-\frac{3}{2}}\right)$ and his derivation is incomplete. It is necessary to prove that $(\tilde{\rho}-1)=o_{p}\left(T^{-\frac{3}{2}}\right)$ to show that the asymptotic power of the DF test is zero, as in this paper. It is impossible to distinguish between the two hypotheses, i.e. " $\rho=1$ " and trend stationary, based on the asymptotic distributions of the OLS estimator. Schmidt and Phillips [14] wrote "they are inconsistent against trend alternatives (West (1987))". We have to be very careful to perform the unit root tests of the random walk with drift.

\subsection{Random Walk Model with Drift and Regression Model with a Constant and a Time Trend}

Finally, I consider the case in which the null hypothesis is the random walk model with drift as given by (8), and the auxiliary regression model contains 
both drift and the time trend:

$$
y_{t}=\beta+\rho y_{t-1}+\delta t+\omega_{t}, y_{0}=0 \text { and } \omega_{0}=0, t=1,2, \cdots, T .
$$

Harvey et al. [27], Elliot et al. [28], and Kwiatkowski et al. [29] considered the estimators of the parameters in the model given by

$$
y_{t}=\beta+\delta t+z_{t}, z_{t}=\rho z_{t}+\varepsilon_{t} .
$$

Models (13) and (14) become the same model if $\rho=1$. The asymptotic distribution of the OLS estimator under (8) is given by (Hamilton [[26], pp. 498-499])

$$
\begin{gathered}
T(\hat{\rho}-1) \stackrel{D}{\longrightarrow} \frac{F}{G}, \\
F=\frac{1}{3}\left(c_{1}-\frac{1}{2} c_{3}\right) d_{1}+\frac{1}{8} d_{2}+\left(c_{3}-\frac{1}{2} c_{1}\right) d_{3}, \\
G=\frac{1}{12} c_{2}-\frac{1}{3} c_{1}^{2}-c_{3}^{2}+c_{1} c_{3}, \\
c_{1}=\int W(r) \mathrm{d} r, c_{2}=\int W(r)^{2} \mathrm{~d} r, c_{3}=\int r W(r) \mathrm{d} r, \\
d_{1}=W(1), d_{2}=W(1)^{2}-1, \text { and } d_{3}=W(1)-\int W(r) \mathrm{d} r
\end{gathered}
$$

The asymptotic distribution of $\tau=(\hat{\rho}-1) /$ s.e. $(\hat{\rho})$ is given by Hamilton [[26], p. 500]. When the true model is the trend stationary model given by (1), the asymptotic distribution of the OLS estimator is given by the following theorem.

$\underline{\text { Theorem } 2.3}$

$$
\tilde{\rho} \stackrel{P}{\longrightarrow} \rho_{0}=\frac{m_{1}}{m_{0}}=r_{1},
$$

where $r_{1}$ is the first order autocorrelation of the error terms. This means that $\tilde{\rho}$ does not converge to 1 , and we can distinguish the random walk with drift from the trend stationary model if the first order correlation of error terms is not equal to 1. Harvey et al. [[27], p. 589] also wrote "each test is applied to data containing either a fixed or local (in sample size) trend, both the asymptotic power and the type I error of the tests rapidly approach zero as the magnitude of the trend increased." However, the present study obtained different results. Only the value of $r_{1}$ does matter and the magnitude of the trend (the value of $\alpha$ ) is irrelevant.

Note that if $\rho=1$ and $\delta \neq 0$, (13) becomes

$$
\Delta y_{t}=\beta+\delta t+\omega_{t} .
$$

This means that $y_{t}$ must be a quadratic function of the time trend, and the model obtained from (17) becomes different from a random walk with drift. As Schmidt and Phillips [[14], p. 258] noted, " $\delta$ represents quadratic trend... This confusion over the meanings of the parameters shows up in properties of the Dickey-Fuller tests...”. The F-test of $H_{0}: \rho=1$ and $\delta=0$ is also used for the unit root tests [30]. This means that $\delta$ should be zero when $\rho$ is 1 and $\rho$ should be less than 1 when $\delta$ is not equal to zero. 


\section{Analysis of US Real GDP}

Figure 1 shows the real US GDP data (billions of chained 2012 dollars, quarterly, seasonally adjusted annual rate from Federal Reserve Economic Data, Economic Research Division, Federal Reserve Bank of St. Louis [31]) from the first quarter of 1950 to the fourth quarter of 2019, i.e., 280 quarters or 70 years. (The 2020 data were not used because the economic impact of COVID-19 is unclear at this time.) The GDP was 2185 billion dollars in the first quarter of 1950, and 19,254 billion dollars in the fourth quarter of 2019. It increased 8.8-fold during the sample period, so $y_{t}=\log _{\mathrm{e}} \mathrm{GDP}_{t}, t=1,2, \cdots, 280$ is used in the analysis. The values $t=1$ and 280 refer to the first quarter of 1950 and the fourth quarter of 2019, respectively. Figure 2 shows the autocorrelation (ACF) and partial autocorrelation (PACF) functions of $y_{t}$. The figure suggests that the process is the AR process (1). All critical values in the first and third cases were obtained from Hamilton [[26], pp. 762-764].

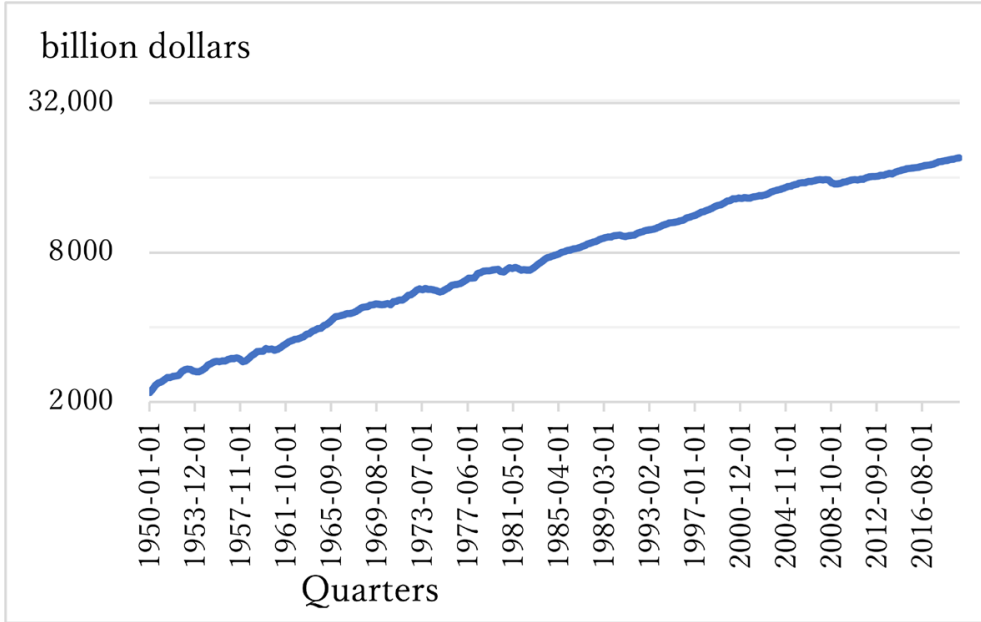

Figure 1. Real US GDP (billions of chained 2012 Dollars, quarterly, seasonally adjusted annual rate) [30].

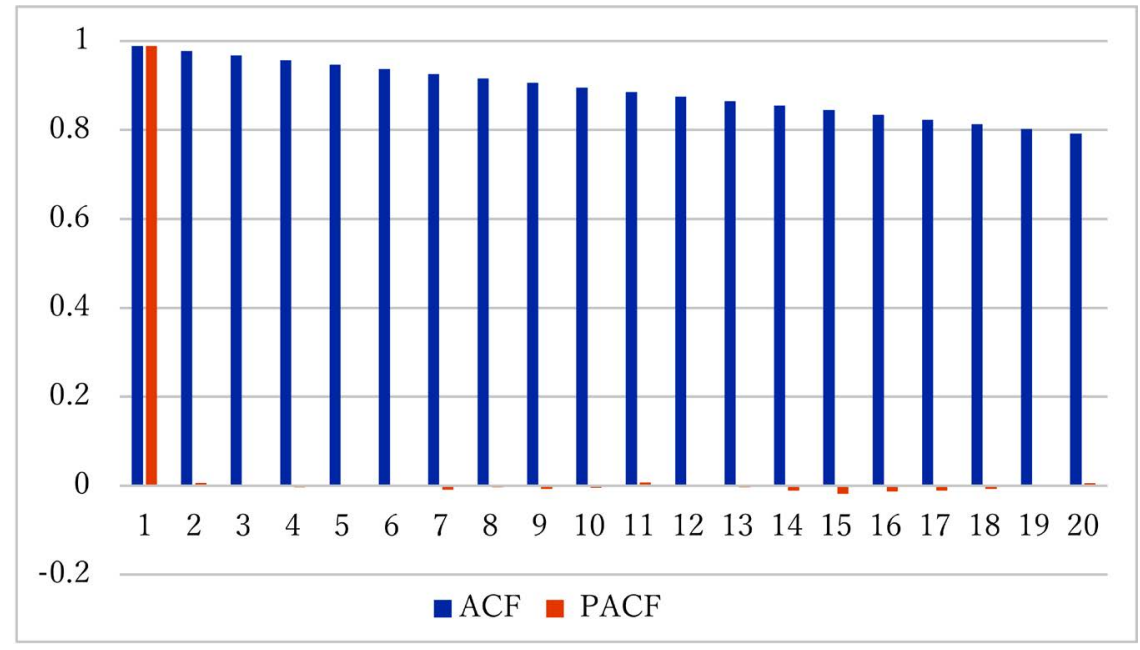

Figure 2. Autocorrelation (ACF) and partial autocorrelation (PACF) functions of $y_{t}$. 


\subsection{Results of the First Case}

In the first case in which the null and auxiliary regression models are given by (3) and (4), the estimated results areas follow (the standard error is given in parentheses):

$$
\begin{aligned}
y_{t}^{*} & =1.00437 y_{t-1}^{*}, R^{2}=0.9997, \\
& (0.000444)
\end{aligned}
$$

$$
T(\hat{\rho}-1)=1.2226 \text { and } \tau=(\hat{\rho}-1) / \text { s.e. }(\hat{\rho})=9.8423 \text {. }
$$

To remove the influence of the initial value, $y_{t}^{*}=y_{t}-y_{0}$ is used in this case. First, $H_{0}: \rho=1$ against $H_{1}: \rho>1$. (Since $y_{t}^{*}$ is clearly an increasing function of $t$, the alternative becomes $\left.H_{1}: \rho>1.\right) T(\hat{\rho}-1)=1.223$ does not exceed the $95 \%$ critical value of 1.28 . However, $\tau$ exceeds the $99 \%$ critical value of 2.01 , which suggests that $\rho>1$. Since the $\tau$ test has usually been used in previous studies, I use the $\tau$ test when the results of the two tests are different. The $95 \%$ confidence interval of $\hat{\rho}$ based on the distribution of $\tau$ is [0.995, 1.011], and $\frac{\rho_{0}}{T}+1=\frac{1.5}{280}+1=1.0054$, obtained from Theorem 2.1, is included in this interval, so the trend stationary alternative is not rejected.

\subsection{Results of the Second Case}

For the second case, the null and auxiliary regression models are given by (8) and (9). The estimation results are

$$
\begin{aligned}
& y_{t}=0.03675+0.9967 y_{t-1}, R^{2}=0.999796, \\
& T^{\frac{3}{2}}(\hat{\rho}-1)=-0.9558, V_{1}=12 m_{0} / \alpha^{2}=0.7203 \text { and } \\
& t=T^{\frac{3}{2}}(\hat{\rho}-1) / \sqrt{V_{1}}=-1.1262 .
\end{aligned}
$$

Therefore, $H_{0}: \rho=1$ against $H_{1}: \rho<1$ is not rejected at the $5 \%$ level. (The $5 \%$ critical value of the standard normal distribution is -1.645$)$. However, in the model trend stationary, $\tilde{\rho}-1=o_{P}\left(T^{-\frac{3}{2}}\right)$ as shown in Theorem 2.2, and we cannot distinguish the random walk with drift model from the trend stationary model based on the distribution of $\hat{\rho}-1$. Therefore, the result may suggest that the model may be either a random walk with drift or trend stationary.

\subsection{Results of the Third Case}

In the third case, the null, alternative and auxiliary regression models are given by (8) and (13). The estimation results are

$$
\begin{gathered}
y_{t}=0.1367+0.9840 y_{t-1}+0.000100 t, R^{2}=0.999798, \\
\quad(0.06573)(0.008394)(0.0000655) \\
T(\hat{\rho}-1)=-4.485 \text { and } \tau=(\hat{\rho}-1) / \text { s.e. }(\hat{\rho})=-1.9083 .
\end{gathered}
$$

The $5 \%$ critical values of the unit root tests based $T(\hat{\rho}-1)$ and $\tau$ are -21.3 
and -3.43 , respectively, and we cannot reject $H_{0}: \rho=1$ against $H_{1}: \rho<1$ at the $5 \%$ level. However, the F-value of $H_{0}: \rho=1$ and $\delta=0$ is 8.3996 , which is bigger than the $5 \%$ critical value of the F-test, 6.34 , and the null hypothesis is rejected at the $5 \%$ level. The following are the results of the DF tests for the first and second cases for $\Delta y_{t}$.

$$
\begin{gathered}
\Delta y_{t}^{*}=-0.37149 \Delta y_{t-1}^{*}, \\
(0.046532) \\
T(\hat{\rho}-1)=-104.0, \tau=(\hat{\rho}-1) / \text { s.e. }(\hat{\rho})=-7.9837, \\
\Delta y_{t}=0.005113+0.35586 \Delta y_{t-1}, R^{2}=0.1284, \\
T^{\frac{3}{2}}(\hat{\rho}-1)=-23.956, V_{1}=12 m_{0} / \alpha^{2}=33.90 \text { and } t=T^{\frac{3}{2}}(\hat{\rho}-1) / \sqrt{V_{1}}=-4.114
\end{gathered}
$$

In these cases, not only the null hypotheses of the unit root but also the trend stationary model are rejected with any reasonable significance level. These results suggest that the process cannot have a quadric trend. In other words, if $\rho=1, \delta$ must be 0 . However, this contradicts the results of the F-test.

\subsection{Trend Stationary Model}

Assuming that $\rho<1$ in (13), we obtain the trend stationary model. The estimates of the trend model are given by

$$
\begin{gathered}
y_{t}=7.8400+0.007738 t, R^{2}=0.9898, \\
(0.00760)(0.00000472)
\end{gathered}
$$

Note that, from Theorem 2.3, we get $\tilde{\rho} \stackrel{P}{\longrightarrow} \rho_{0}=0.9896$, very close to 1 . Let $e_{t}$ be the residuals of Equation (22) (detrended values of $y_{t}$ ). Figure 3 shows ACF and partial PACF of $e_{t}$. The figure strongly implies that $e_{t}$ follows the $\operatorname{AR}(p)$, and the value of $p$ is small. We can apply the $\mathrm{ADF}$ test [2] to $e_{t}$. The results are as follows (the model is selected by the Schwarz Bayes information criterion):

$$
\begin{gathered}
e_{t}=\gamma_{1} e_{t-1}+\gamma_{2} \Delta e_{t-1}=-0.01813 e_{t-1}+0.3625 \Delta e_{t-1}, R^{2}=0.9821, \\
(0.05543) \quad(0.05527)
\end{gathered}
$$

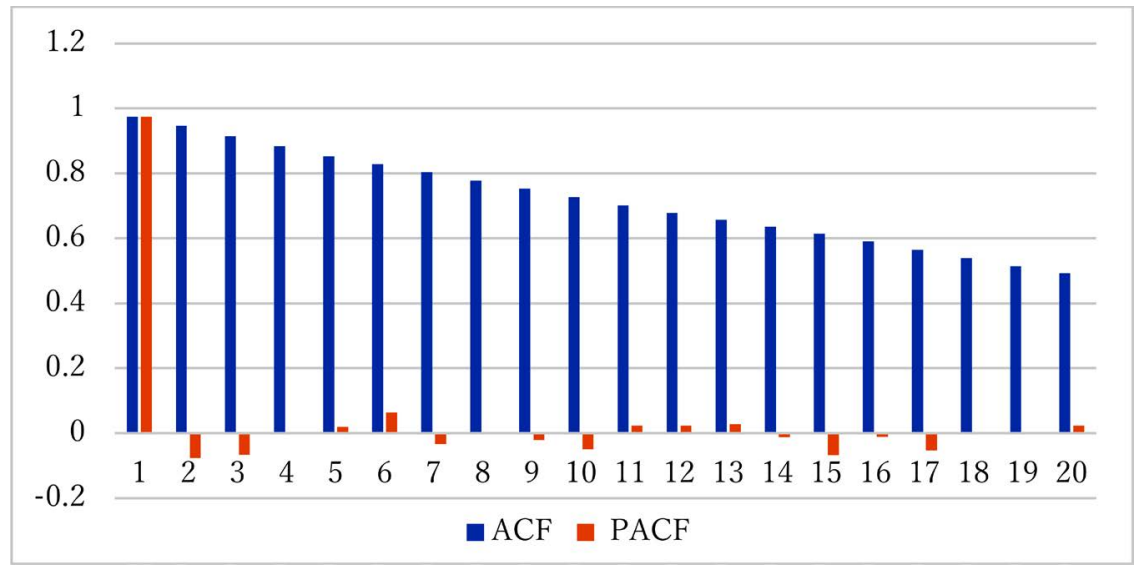

Figure 3. Autocorrelation (ACF) and partial autocorrelation (PACF) functions of $e_{t}$. 


$$
\tau=\left(\hat{\gamma}_{1}-1\right) / \text { s.e. }\left(\hat{\gamma}_{1}\right)=-2.2688 .
$$

The $5 \%$ critical value of $\tau$ is -1.95 , and we can reject the null hypothesis of the unit root for the detrended process; the trend stationary assumption is accepted. The result is very close to the unit root process, and it is consistent with previous studies; some found a unit root and others did not. When the process is trend stationary, the estimator of $\alpha$ becomes a supper efficient estimator, and we can use the ADF test for the residuals.

\subsection{Economic Implications}

Since the trend stationary model was supported in the previous section, we combine (22) and (23) and consider the model given by:

$$
\hat{y}_{t}=7.8400+0.007738 t+1.3443 e_{t-1}-0.3625 e_{t-2}
$$

$R^{2}$ obtained from $\hat{y}_{t}$ is 0.99979 . The very large $R^{2}$ indicates that $\hat{y}_{t}$ fits $y_{t}$ very well.

Using (24), we can simulate an economic shock to the economy in a given period (for example, the Lehman shock in 2008). Figure 4 shows the impacts of the negative shock. The shock is normalized as -1 and occurs at time zero. The impacts are larger than the original shock for three quarters, peaking at $45 \%$ larger than the original shock, and then gradually declines, showing a cyclical movement [32]. The impact continues for a long period, dropping to half the original impact even after 40 quarters or 10 years. This finding may have important implications for policy makers. The world economy is now facing a serious problem due to COVID-19 and may suffer a long-term depression unless proper economic countermeasures are taken.

\section{Conclusions}

In this paper, I considered the asymptotic properties of the OLS estimator when the true model is trend stationary. The power of the DF depends on the null and auxiliary regression models. I considered the following three cases: 1) the null model is a random walk without drift and the auxiliary regression model does

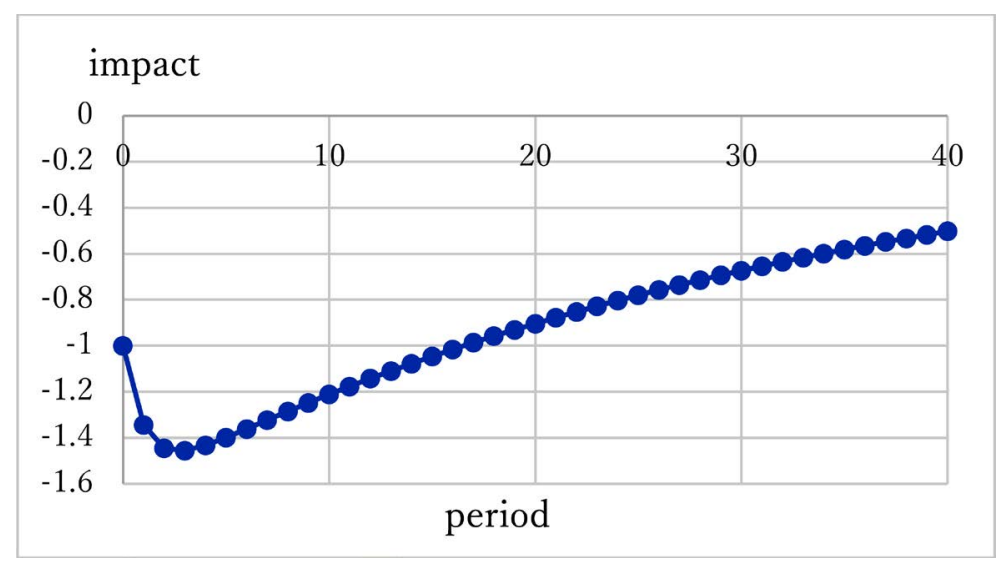

Figure 4. Impacts of economic shock $(-1$ at $t=0)$. 
not contain a constant; 2) the null model is a random walk with drift and the auxiliary regression model contains a constant; and 3) the null model is a random walk with drift and the auxiliary regression model contains both a constant and a time trend. Although the DF test has no asymptotic power for the second case, we can distinguish between the random walk and trend stationary models in first and third cases in contrast to what was suggested in previous studies. The asymptotic distribution of the OLS estimator depends on the first order of the autocorrelation of error terms in the third case.

Then I analyzed the real US GDP for 280 quarters or 70 years. The trend stationary model with AR (2) errors fits very well and gives a very large $R^{2}$ value. However, the result is very close to the unit root process and is consistent with those of previous studies, some of which found a unit root while others did not. Then the impacts of the economic shock (such as the Lehman stock) were evaluated. The impacts were found to continue for a long period and to take 40 quarters or 10 years to decrease to half the original impact.

In this study, only the real US GDP was analyzed. Economic variables such as the GDP of various countries will be studied in the future to determine whether they follow the random walk model or trend model.

\section{Conflicts of Interest}

The author declares no conflicts of interest regarding the publication of this paper.

\section{References}

[1] Dickey, D.A.I. and Fuller, W.A. (1979) Distribution of the Estimators for Autoregressive Time Series with a Unit Root. Journal of the American Statistical Association, 74, 427-431. https://doi.org/10.1080/01621459.1979.10482531

[2] Said, E. and Dickey, D.A.I. (1984) Testing for Unit Roots in Autoregressive-Moving Average Models of Unknown Order. Biometrika, 71, 599-607. https://doi.org/10.1093/biomet/71.3.599

[3] Perron, P. (1989) The Great Crash, the Oil Price Shock, and the Unit Root Hypothesis. Econometrica, 57, 1391-1401. https://doi.org/10.2307/1913712

[4] Dolado, J.J., Gonzalo, J. and Mayoral, L. (2002) A Fractional Dickey-Fuller Test for Unit Roots. Econometrica, 70, 1963-2006. https://doi.org/10.1111/1468-0262.00359

[5] Leybourne, S., Kim, T.H. and Newbold, P. (2005) Examination of Some More Powerful Modifications of the Dickey-Fuller Test. Journal of Time Series Analysis, 26, 355-369. https://doi.org/10.1111/j.1467-9892.2004.00406.x

[6] Hacker, R.S. and Hatemi, A.-J. (2010) The Properties of Procedures Dealing with Uncertainty about Intercept and Deterministic Trend in Unit Root Testing. CESIS Electronic Working Paper Series, Paper No. 214, Centre of Excellence for Science and Innovation Studies (CESIS), Royal Institute of Technology, Stockholm.

[7] Casini, A. and Perron, P. (2018) Structural Breaks in Time Series. https://doi.org/10.1093/acrefore/9780190625979.013.179

[8] Nagai, K., Nishiyama, Y. and Hitomi, K. (2018) Sequential Test for Unit Root in AR(1) Model. KIER Discussion Paper No.1003, Kyoto University, Kyoto. 
[9] Paparoditis, E. and Politis, D.N. (2018) The Asymptotic Size and Power of the Augmented Dickey_Fuller Test for a Unit Root. Econometric Reviews, 37, 955-973. https://doi.org/10.1080/00927872.2016.1178887

[10] Muthuramu, P. and Uma Maheswari, T. (2019) Tests for Structural Breaks in Time Series Analysis: A Review of Recent Development. Shanlax International Journal of Economics, 7, 66-79. https://doi.org/10.34293/economics.v7i4.628

[11] Enders, W. (2015) Applied Econometric Time Series. 4th Edition, John Wiley \& Sons, Hoboken.

[12] Haldrup, L. and Jansson, N.M. (2006) Improving Size and Power in Unit Root Testing. In: Miller, T.C. and Patterson, K., Eds., Palgrave Handbook of Econometrics, Vol.1, Palgrave Macmillan, London. https://dx.doi.org/10.2139/ssrn.1147594

[13] West, K.D. (1987) A Note on the Power of Least Squares Tests for a Unit Root. Economics Letters, 24, 249-252. https://doi.org/10.1016/0165-1765(87)90125-X

[14] Schmidt, P. and Phillips, P.C.B. (1992) LM Tests for a Unit Root of the Deterministic Trends. Oxford Bulletin of Economics and Statistics, 54, 257-287. https://doi.org/10.1111/j.1468-0084.1992.tb00002.x

[15] Fleissig, A.R. and Strauss, J. (1999) Is OECD Real Per Capita GDP Trend or Difference Stationary? Evidence from Panel Unit Root Tests. Journal of Macroeconomics, 21, 673-690. https://doi.org/10.1016/S0164-0704(99)80001-9

[16] Hegwood, N. and Papell, D.H. (2007) Are Real GDP Levels Trend, Difference, or Regime-Wise Trend Stationary? Evidence from Panel Data Tests Incorporating Structural Change. Southern Economic Journal, 74, 104-113.

[17] Ozturk, I. and Kalyoncu, H. (2007) Is Per Capita Real GDP Stationary in the OECD Countries? Evidence from a Panel Unit Root Test. Ekonomski Pregled, 58, 680-688.

[18] Cuestas, J.C. and Garratt, D. (2011) Is Real GDP Per Capita a Stationary Process? Smooth Transitions, Nonlinear Trends and Unit Root Testing. Empirical Economics, 41, 555-563. https://doi.org/10.1007/s00181-010-0389-0

[19] Furuoka, F. (2011) Is GDP in ASEAN Countries Stationary? New Evidence from Panel Unit Root Tests. Economics Bulletin, 31, 1391-1400.

[20] Charles, A. and Darné, O. (2012) Trends and Random Walks in Macroeconomic Time Series: A Reappraisal. Journal of Macroeconomics, 34, 167-180. https://doi.org/10.1016/j.jmacro.2011.10.001

[21] Lee, K.C. (2014) Is Per Capita Real GDP Stationary in China? Sequential Panel Selection Method. Economic Modelling, 37, 507-517.

https://doi.org/10.1016/j.econmod.2013.11.040

[22] Oskooe, S.A.P. and Akbari, L.T. (2015) Is Per Capita Real GDP Stationary? Evidence from OPEC Countries. International Journal of Humanities and Social Science, 5, 166-168.

[23] Xing, L.Z., Dong, X.L. and Guan, J. (2017) Global Industrial Impact Coefficient Based on Random Walk Process and Inter-Country Input-Output Table. Physica A: Statistical Mechanics and its Applications, 471, 576-591. https://doi.org/10.1016/j.physa.2016.12.070

[24] Zeren, F. and İşlek, H. (2019) Is Per Capita Real GDP Stationary in the D-8 Countries? Evidence from a Panel unit Root Test. In: Akay, E.Ç. and Korkmaz, Ö., Eds., Selected Topics in Applied Econometrics, Peter Lang, Pieterlen and Bern, 67-86.

[25] Phillips, P.C.B. and Perron, P. (1988) Testing for a Unit Root in Time Series Regression. Biometrika, 75, 335-346. https://doi.org/10.1093/biomet/75.2.335

[26] Long, Z.M. and Herrera, R. (2020) Spurious OLS Estimators of Detrending Method 
by Adding a Linear Trend in Difference-Stationary Processes-A Mathematical Proof and Its Verification by Simulation. Mathematics, 8, 1931.

https://doi.org/10.3390/math8111931

[27] Hamilton, J.D. (1994) Time Series Analysis. Princeton University Press, Princeton. https://doi.org/10.1515/9780691218632

[28] Harvey, D.I., Leybourne, S.J. and Taylor, A.M.B. (2009) Unit Root Testing in Practice: Dealing with Uncertainty over the Time Trend and Initial Conditions. Econometric Theory, 25, 587-636. https://doi.org/10.1017/S026646660809018X

[29] Elliot, G., Rothenberg, T.J. and Stock, J.H. (1996) Efficient Tests for an Autoregressive Unit Root. Econometrica, 64, 813-836. https://doi.org/10.2307/2171846

[30] Kwiatkowski, D., Phillips, P.C.B., Schmidt, P. and Shin, Y. (1992) Testing the Null Hypothesis of Stationarity against the Alternative of a Unit Root: How Sure Are We That Economic Time Series Have a Unit Root? Journal of Econometrics, 54, 159-178. https://doi.org/10.1016/0304-4076(92)90104-Y

[31] Dickey, D.A.I. and Fuller, W.A. (1981) Likelihood Ratio Statistics Four Autoregressive Time Series with a Unit Root. Econometrica, 49, 1057-1072.

https://doi.org/10.2307/1912517

[32] Economic Research Division, Federal Reserve Bank of St. Louis Federal Reserve (2020) Economic Data. https://fred.stlouisfed.org 


\section{Appendix A1. Proof of Theorem 2.1}

When the alternative model given by (1) is the true one, the OLS estimator becomes

$$
\begin{gathered}
\tilde{\rho}=\frac{\sum_{t=1}^{T} y_{t} y_{t-1}}{\sum_{t=1}^{T} y_{t-1}^{2}}=1+\frac{\sum_{t=1}^{T} y_{t-1}\left(y_{t}-y_{t-1}\right)}{\sum_{t=1}^{T} y_{t-1}^{2}} \\
=1+\frac{\sum_{t=1}^{T}\left\{\alpha(t-1)+u_{t-1}\right\}\left(\alpha+u_{t}-u_{t-1}\right)}{\sum_{t=1}^{T}\left\{\alpha(t-1)+u_{t-1}\right\}^{2}} \\
\tilde{\rho}-1=\frac{\sum_{t=1}^{T}\left\{\alpha(t-1)+u_{t-1}\right\}\left(\alpha+u_{t}-u_{t-1}\right)}{\sum_{t=1}^{T}\left\{\alpha(t-1)+u_{t-1}\right\}^{2}}=\frac{A_{T}}{B_{T}} .
\end{gathered}
$$

For the numerator,

$$
\begin{aligned}
A_{T}= & \sum_{t=1}^{T} \alpha^{2}(t-1)+\alpha \sum_{t=1}^{T} u_{t-1}+\sum_{t=1}^{T} \alpha(t-1)\left(u_{t}-u_{t-1}\right) \\
& +\sum_{t=1}^{T} u_{t-1} u_{t}-\sum_{t=1}^{T} u_{t-1}^{2} \\
\equiv & \alpha^{2} \frac{T(T-1)}{2}+A_{1}+A_{2}+A_{3}+A_{4} .
\end{aligned}
$$

It is easy to show that

$$
A_{1}=O_{p}(T) \text { and } A_{4}=O_{p}(T)
$$

Here,

$$
\begin{aligned}
A_{2} / \alpha= & \left(u_{2}-u_{1}\right)+2\left(u_{3}-u_{2}\right)+\cdots+(T-2)\left(u_{T-1}-u_{T-2}\right) \\
& +(T-1)\left(u_{T}-u_{T-1}\right) \\
= & -\left(u_{1}+u_{2}+\cdots+u_{t-1}\right)+(T-1) u_{T} \\
= & (T-1) u_{T}+O_{p}\left(T^{\frac{1}{2}}\right)=O_{p}(T) .
\end{aligned}
$$

Since $E\left(A_{3}\right)=\sigma^{2} m_{1} T$ and the fourth moment of $u_{t}$ is finite,

$$
A_{3}=O_{P}(T) \text {. }
$$

From (A.2)-(A.5),

$$
\frac{A_{T}}{T^{2}} \stackrel{P}{\longrightarrow} \frac{\alpha^{2}}{2}
$$

For the denominator,

$$
\begin{aligned}
B_{T} & =\sum_{t=1}^{T}\left\{\alpha^{2}(t-1)^{2}+2 \alpha(t-1) u_{t-1}+u_{t-1}^{2}\right\} \\
& =\alpha^{2} \frac{T(T-1)(2 T-1)}{6}+2 \sum_{t=1}^{T} \alpha(t-1) u_{t-1}+\sum_{t=1}^{T} u_{t-1}^{2} \\
& \equiv \alpha^{2} \frac{T(T-1)(2 T-1)}{6}+B_{1}-A_{4} .
\end{aligned}
$$

Here,

$$
E\left\{\sum_{t=1}^{T}(t-1) u_{t-1}\right\}=0 \text { and } \sum_{s=1}^{\infty}\left|m_{s}\right| s^{n}<\infty
$$


and

$$
\begin{aligned}
& E\left\{\sum_{t=1}^{T}(t-1) u_{t-1}\right\}^{2} \\
& =\sum_{t=1}^{T}(t-1)^{2}+2 \sum_{i=1}^{T} \sum_{j=i+1}^{T}(i-1)(j-1) E\left(u_{i-1} u_{j-1}\right) \\
& =m_{0} \sum_{t=1}^{T}(t-1)^{2}+\sum_{i=1}^{T} \sum_{j=i+1}^{T}(i-1)(i-1+j-i) m_{j-i} \\
& =m_{0} \sum_{t=1}^{T}(t-1)^{2}+\sum_{i=1}^{T}(i-1)^{2} \sum_{s=1}^{T-i} m_{s}+\sum_{t=1}^{T}(i-1) \sum_{s=1}^{T-i} s m_{s} \\
& <m_{0} \frac{T(T-1)(2 T-1)}{6}+\varphi_{1} \frac{T(T-1)(2 T-1)}{6}+\varphi_{2} \frac{T(T-1)}{2}=O\left(T^{3}\right),
\end{aligned}
$$

where $\varphi_{1}=\sum_{s=1}^{\infty}\left|m_{s}\right|$ and $\varphi_{2}=\sum_{s=1}^{\infty}\left|s m_{s}\right|$

From (A.8) and (A.9), we get

$$
B_{1}=O_{p}\left(T^{3 / 2}\right) .
$$

From (A.3), (A.7), and (A.10),

$$
\frac{B_{T}}{T^{3}} \stackrel{P}{\longrightarrow} \frac{\alpha^{2}}{3} .
$$

From (A.1), (A.7), (A.11), and Slutsky's theorem,

$$
T(\tilde{\rho}-1) \stackrel{P}{\longrightarrow} \frac{3}{2} \text {. }
$$

\section{Appendix A2. Proof of Theorem 2.2}

Here, $y_{t}-y_{t-1}=\alpha+u_{t}-u_{t-1}$ and the OLS estimator of $\rho$ becomes

$$
\begin{gathered}
\tilde{\rho}=\frac{\sum_{t=1}^{T}\left(y_{t-1}-\bar{y}_{1}\right)\left(y_{t}-\bar{y}\right)}{\sum_{t=1}^{T}\left(y_{t-1}-\bar{y}_{1}\right)^{2}}, \\
\tilde{\rho}-1=\frac{\sum_{t=1}^{T}\left(y_{t-1}-\bar{y}_{1}\right)\left(y_{t}-\bar{y}-y_{t-1}+\bar{y}_{1}\right)}{\sum_{t=1}^{T}\left(y_{t-1}-\bar{y}_{1}\right)^{2}} \\
=\frac{\sum_{t=1}^{T}\left(y_{t-1}-\bar{y}_{1}\right)\left(y_{t}-y_{t-1}+\bar{y}_{1}-\bar{y}_{2}\right)}{\sum_{t=1}^{T}\left(y_{t-1}-\bar{y}_{1}\right)^{2}} \\
=\frac{\sum_{t=1}^{T}\left(y_{t-1}-\bar{y}_{1}\right)\left(\alpha+u_{t}-u_{t-1}+\bar{y}_{1}-\bar{y}\right)}{\sum_{t=1}^{T}\left(y_{t-1}-\bar{y}_{1}\right)^{2}} \\
\equiv \frac{C_{T}}{D_{T}},
\end{gathered}
$$

$\bar{y}=\frac{1}{T} \sum_{t=1}^{T} y_{t}$, and $\bar{y}_{1}=\frac{1}{T} \sum_{t=1}^{T} y_{t-1}$.

For the numerator, since $\sum_{t=1}^{T}\left(y_{t-1}-\bar{y}_{1}\right)=0$ and $\bar{y}_{1}-\bar{y}=\frac{y_{T}}{T}$, we get

$$
\begin{aligned}
C_{T} & =\sum_{t=1}^{T}\left(y_{t-1}-\bar{y}_{1}\right)\left(u_{t}-u_{t-1}\right) \\
& =\sum_{t=1}^{T} y_{t-1}\left(u_{t}-u_{t-1}\right)+\bar{y}_{1} \sum_{t=1}^{T}\left(u_{t}-u_{t-1}\right) \\
& =\sum_{t=1}^{T} y_{t-1}\left(u_{t}-u_{t-1}\right)+\bar{y}_{1} u_{T}
\end{aligned}
$$




$$
\begin{aligned}
& =\sum_{t=1}^{T}\left(\alpha(t-1)+u_{t-1}\right)\left(u_{t}-u_{t-1}\right)+\bar{y}_{1} u_{T} \\
& =\sum_{t=1}^{T} \alpha(t-1)\left(u_{t}-u_{t-1}\right)+\sum_{t=1}^{T} u_{t-1} u_{t}-\sum_{t=1}^{T} u_{t-1}^{2}+\bar{y}_{1} u_{T} \\
& =A_{2}+A_{3}+A_{4}+\bar{y}_{1} u_{T} .
\end{aligned}
$$

As before, $A_{2}=O_{p}(T), A_{3}=O_{P}(T)$ and $A_{4}=O_{p}(T)$.

Here,

$$
\begin{aligned}
\bar{y}_{1} & =\frac{1}{T} \sum_{t=1}^{T} \alpha(t-1)+\frac{1}{T} \sum_{t=1}^{T} u_{t} \\
& =\alpha \frac{T-1}{2}+\frac{1}{T} \sum_{t=1}^{T} u_{t} \\
& =\alpha \frac{T-1}{2}+O_{p}\left(T^{-\frac{1}{2}}\right),
\end{aligned}
$$

and $\bar{y}_{1} u_{T}=O_{p}(T)$.

From (A.14) and (A.15),

$$
\frac{C_{T}}{T} P \stackrel{P}{\longrightarrow} \frac{\alpha}{2} u_{T}+m_{0} .
$$

For the denominator,

$$
D_{T}=\sum_{t=1}^{T} y_{t-1}^{2}-T \bar{y}_{1}^{2} \equiv D_{1}+D_{2} .
$$

From (A.10)

$$
\begin{aligned}
D_{1} & =\sum_{t=1}^{T}\left\{\alpha(t-1)+u_{t-1}\right\}^{2} \\
& =\alpha^{2} \sum_{t=1}^{T}(t-1)^{2}+2 \sum_{t=1}^{T}(t-1) u_{t-1}+\sum_{t=1}^{T} u_{t-1}^{2} \\
& =\frac{\alpha^{2} T(T-1)(2 T-1)}{6}+O_{P}\left(T^{3 / 2}\right) .
\end{aligned}
$$

From (A.18),

$$
D_{2}=-\frac{\alpha^{2} T(T-1)^{2}}{4}+O_{P}\left(T^{\frac{1}{2}}\right) .
$$

From (A.17)-(A.19),

$$
D_{T}=\frac{\alpha^{2} T^{3}}{12}+O_{P}\left(T^{2}\right)
$$

From (A13), (A16) and (A.20), we get

$$
T^{2}(\tilde{\rho}-1) \stackrel{p}{\longrightarrow} \frac{6}{\alpha} u_{T}+\frac{12}{\alpha^{2}} m_{0} \text { and } T^{2}(\tilde{\rho}-1) \stackrel{D}{\longrightarrow} N\left(\frac{12}{\alpha^{2}} m_{0}, \frac{36}{\alpha^{2}} m_{0}\right) .
$$

A.3 Proof of Theorem 2.3

The OLS estimator of $\rho$ is equivalent to the OLS estimator of

$$
\begin{gathered}
y_{t}^{*}=\rho y_{t-1}^{+}+\delta t^{*}+u_{t}^{*} \\
y_{t}^{*}=y_{t}-\bar{y}, y_{t-1}^{+}=y_{t-1}-\bar{y}_{1}, t^{*}=t-\bar{T}, \bar{T}=\frac{1}{T} \sum_{t=1}^{T} t=\frac{T+1}{2}, \\
u_{t}^{*}=u_{t}-\bar{u} \text { and } \bar{u}=\frac{1}{T} \sum_{t=1}^{T} u_{t} .
\end{gathered}
$$


Let

$$
y_{t}^{*}=a t^{*}+v_{t} .
$$

Then the OLS estimator of $a$ is given by

$$
\begin{gathered}
\hat{a}=\frac{\sum_{t=1}^{T} t^{*} y_{t}^{*}}{\sum_{t}^{T} t^{* 2}}=\frac{\sum_{t=1}^{T} t^{*} y_{t}}{\sum_{t}^{T} t^{* 2}}=\frac{\sum_{t=1}^{T} t^{*}\left(\alpha t+u_{t}\right)}{\sum_{t}^{T} t^{* 2}}=\alpha+\frac{\sum_{t=1}^{T} t^{*} u_{t}}{\sum_{t}^{T} t^{* 2}} \\
\varsigma=\hat{a}-\alpha \sim N\left(0, \frac{m_{0}}{\sum_{t=1}^{T} t^{* 2}}\right), \sum_{t=1}^{T} t^{* 2}=\frac{T(T+1)(T-1)}{12}, \text { and } \\
T^{\frac{3}{2}} \varsigma D \stackrel{D}{\longrightarrow} N\left(0,12 m_{0}\right) .
\end{gathered}
$$

In the same way, let

$$
y_{t-1}^{+}=b t^{*}+w_{t} .
$$

Then the OLS estimator of $b$ is given by

$$
\begin{gathered}
\hat{b}=\frac{\sum_{t=1}^{T} t^{*} y_{t}^{+}}{\sum_{t}^{T} t^{* 2}}=\frac{\sum_{t=1}^{T} t^{*} y_{t-1}}{\sum_{t}^{T} t^{* 2}}=\frac{\sum_{t=1}^{T} t^{*}\left\{\alpha(t-1)+u_{t-1}\right\}}{\sum_{t}^{T} t^{* 2}} \\
=\alpha+\frac{-\sum_{t=1}^{T} t^{*} \alpha+\sum_{t=1}^{T} t^{*} u_{t-1}}{\sum_{t}^{T} t^{* 2}}, \\
\eta=\hat{b}-\alpha=\frac{-\sum_{t=1}^{T} t^{*} \alpha+\sum_{t=1}^{T} t^{*} u_{t-1}}{\sum_{t}^{T} t^{* 2}}, \\
E(\eta)=E(\hat{b})-\alpha=\frac{-\alpha \sum_{t=1}^{T} t^{*}}{\sum_{t=1}^{T} t^{* 2}}=0, \text { and } \\
V(\eta)=\frac{\sigma^{2} \sum_{t=2}^{T} t^{* 2}}{\left\{\sum_{t=1}^{T} t^{* 2}\right\}^{2}}=\frac{T^{-3}}{12}+O\left(T^{-4}\right), \eta=O_{p}\left(T^{-3 / 2}\right) .
\end{gathered}
$$

Let

$$
\begin{aligned}
\hat{v}_{t}=y_{t}^{*}- & \hat{a} t^{*}=y_{t}-\bar{y}-(\alpha+\varsigma)(t-\bar{T})=u_{t}-\bar{u}-\zeta t^{*}, \text { and } \\
\hat{w}_{t} & =y_{t-1}^{+}-\hat{b} t^{*}=y_{t-1}-\bar{y}_{1}-\hat{b} t^{*} \\
& =\alpha(t-1)+u_{t-1}-\alpha \bar{T}_{1}-\bar{u}_{1}-(\alpha+\eta)(t-\bar{T}) \\
& =-\alpha+u_{t-1}+\alpha\left(\bar{T}-\bar{T}_{1}\right)-\bar{u}_{1}-\eta(t-\bar{T}) \\
& =u_{t-1}-\bar{u}_{1}-\eta t^{*} .
\end{aligned}
$$

Then the OLS estimator of $\rho$ in (A.22) becomes

$$
\tilde{\rho}=\frac{\sum_{t=1}^{T} \hat{v}_{t} \hat{w}_{t}}{\sum_{t=1}^{T} \hat{w}^{2}} \equiv \frac{H_{T}}{I_{T}} .
$$

For the numerator,

$$
H_{T}=\sum_{t=1}^{T} \hat{v}_{t} \hat{w}_{t}=\sum_{t=1}^{T}\left(u_{t}-\bar{u}-\zeta t^{*}\right)\left(u_{t-1}-\bar{u}_{1}-\eta t^{*}\right) .
$$


Since $\bar{u}=O_{p}\left(T^{-\frac{1}{2}}\right), \bar{u}_{1}=O_{p}\left(T^{-\frac{1}{2}}\right)$ and $\zeta=O_{p}\left(T^{-3 / 2}\right)$,

$$
\begin{gathered}
H_{T}=\sum_{t=1}^{T} \hat{v}_{t} \hat{w}_{t}=\sum_{t=1}^{T}\left(u_{t} u_{t-1}\right)-T \eta \sum_{t=1}^{T} \frac{1}{T} t^{*} u_{t}+O_{p}\left(T^{1 / 2}\right), \text { and } \\
T^{-1} H_{T} \stackrel{D}{\longrightarrow} T^{-1} \sum_{t=1}^{T}\left(u_{t} u_{t-1}+\frac{3 \alpha}{2} \lambda_{t} u_{t}\right), \lambda_{t}=\frac{t^{*}}{T} .
\end{gathered}
$$

From the assumption of (2) $V\left(\sum_{t=1}^{T} u_{t} u_{t-1}\right)=O(T)$. Therefore,

$$
T^{-1} \sum_{t=1}^{T} u_{t} u_{t-1} \stackrel{P}{\longrightarrow} m_{1} \text { and } T^{-1} \sum_{t=1}^{T} u_{t} \lambda_{t} \stackrel{P}{\longrightarrow} 0 \text {. }
$$

For the denominator,

$$
\begin{aligned}
I_{T}= & \sum_{t=1}^{T} \hat{w}^{2}=\sum_{t=1}^{T}\left(u_{t-1}-\bar{u}_{1}-\eta t^{*}\right)^{2} \\
= & \sum_{t=1}^{T} u_{t-1}^{2}+\eta^{2} \sum_{t=1}^{T} t^{* 2}+T \bar{u}_{1}^{2}-2 \eta \sum_{t=1}^{T} t^{*} u_{t-1} \\
& -2 \bar{u}_{1} \sum_{t=1}^{T} u_{t-1}+2 \eta \bar{u}_{1} \sum_{t=1}^{T} t^{*}
\end{aligned}
$$

Since $\sum_{t=1}^{T} t^{*} u_{t-1}=O_{p}\left(T^{\frac{3}{2}}\right), \eta=O_{p}\left(T^{-3 / 2}\right), \bar{u}_{1}=O_{p}\left(T^{-\frac{1}{2}}\right)$ and $\eta=O_{p}\left(T^{-1}\right)$,

$$
I_{T}=\sum_{t=1}^{T} u_{t-1}^{2}+O_{P}\left(T^{\frac{1}{2}}\right) .
$$

Since $\sum_{t=1}^{T} u_{t-1}^{2} / T \stackrel{P}{\longrightarrow} m_{0}$,

$$
\stackrel{I_{T}}{T} \stackrel{P}{\longrightarrow} m_{0} .
$$

From (A.28), (A.31), and (A.34), we get

$$
\tilde{\rho} \stackrel{P}{\longrightarrow} \frac{m_{1}}{m_{0}}=r_{1} .
$$

